November 9, 2018 11:44 WSPC/INSTRUCTION FILE iHMC-ijmpc-rev

International Journal of Modern Physics C

(C) World Scientific Publishing Company

\title{
PROPERTIES OF ITERATIVE MONTE CARLO SINGLE HISTOGRAM REWEIGHTING
}

\author{
MARTIN GMITRA \\ Department of Theoretical Physics and Astrophysics, P.J.Šafárik University, \\ Park Angelinum 9, 04001 Košice, Slovak Republic \\ gmitra@kosice.upjs.sk \\ DENIS HORVÁTH \\ Department of Theoretical Physics and Astrophysics, P.J.Šafárik University, \\ Park Angelinum 9, 04001 Košice, Slovak Republic \\ horvath.denis@gmail.com \\ Received Day Month Year \\ Revised Day Month Year
}

\begin{abstract}
We present iterative Monte Carlo algorithm for which the temperature variable is attracted by a critical point. The algorithm combines techniques of single histogram reweighting and linear filtering. The $2 \mathrm{~d}$ Ising model of ferromagnet is studied numerically as an illustration. In that case, the iterations uncovered stationary regime with invariant probability distribution function of temperature which is peaked nearly the pseudocritical temperature of specific heat. The sequence of generated temperatures is analyzed in terms of stochastic autoregressive model. The error of histogram reweighting can be better understood within the suggested model. The presented model yields a simple relation, connecting variance of pseudocritical temperature and parameter of linear filtering.
\end{abstract}

Keywords: Monte Carlo; histogram reweighting; autoregressive model

PACS Nos.: 02.70.Tt, 05.50.+q, 02.50.Ey, 05.65.+b

\section{Introduction}

The critical phenomena, typical of many body systems, have attracted remarkable scientific interest since a long time. The Monte Carlo (MC) stochastic techniques 1 serve to estimate thermodynamic averages from samples of the configuration space. Many advanced simulation approaches are rooted in elementary updates of Metropolis algorithm 2, which generates a Markovian chain of spin configurations.

Most of the efforts try to improve MC method within the critical region. The efficiency of MC method have been increased by the development of sampling and data processing techniques. The reweighting technique applied anew to classical statistical systems 344 enables to perform a random sampling for one distribution function, and to calculate quantities of interest for similar distributions. To be more 
concrete, let us assume that a sample of $N$ configurations $(q=1,2, \ldots, N)$ of quantity $Q$ have been accumulated for a fixed temperature $T_{t}$. The samples $Q_{q}$ are used to construct a continuous temperature $T$ dependence of a single histogram approximation $\langle Q\rangle_{T, T_{t}}$ of the canonical thermodynamic average $\langle Q\rangle_{T}$. The interpolation called "reweighting on the fly" $[5$ is based on the formula

$$
\langle Q\rangle_{T, T_{t}}=\frac{\sum_{q=1}^{N} Q_{q} \exp \left[\frac{E_{q}}{T_{t}}\left(1-\frac{T_{t}}{T}\right)\right]}{\sum_{q=1}^{N} \exp \left[\frac{E_{q}}{T_{t}}\left(1-\frac{T_{t}}{T}\right)\right]},
$$

where $E_{q}$ is the energy (in Boltzmann constant units) of configuration $q$. Because of the finite length of the MC run, Eq. (1) provides reliable results only for a relatively narrow range of $T$ values around $T_{t}$. When $T$ differs too much from $T_{t}$, it causes an increase of the statistical errors and may lead to unreliable results. The reliable range of $T$ values also decreases as the system size increases 67 . A qualitative study in Ref. 8 has shown that reweighting causes a systematic shift in the height and the location of the specific heat peak, which depends on the relative position of $T_{t}$ and $T$. It turns out that the shift decreases as $\sim N^{-1 / 2}$ only for $N$ much larger than the number of degrees of freedom. Systematic errors, eventually affected by sampling autocorrelations, may also take place at finite $N$. The first quantitative study of the statistical errors in reweighted data $[5$ has demonstrated that theoretical calculation of the error is both difficult and time consuming so it may not be justified for all studies. In this paper we present rather general iterative treatment based on the single histogram reweighting which implicitly takes into account both the systematic errors of reweighting and the statistical errors to generate stochastic fluctuations near a critical point.

A well known systematic error, present in the histogram method, is an overestimation (underestimation) of $\langle E\rangle_{T, T_{t}}$ when $T \ll T_{t}\left(T \gg T_{t}\right)$, respectively 6 . This fact and the general idea of feedback regulation $\frac{9}{}$ are used to construct an iterative algorithm. The aim of the suggested method is to estimate the critical temperature or other parameters of interest, as well as to perform an analysis and partial elimination of their errors. The method combines single reweighting, linear filtering and an iterative treatment. The incorporation of iterations is inspired by a general class of probabilistic models 10. The iterations generate data that are further analyzed in terms of a suggested autoregressive moving average (ARMA) model.

The single-spin flip Metropolis algorithm is applied here in order to obtain averages for moderate lattice sizes. The cluster methods are not used due to the efficiency reasons discussed in Ref. 11. The efficiency of the algorithm should also be increased by hybrid schemes 12 . However, because of many side effects that can show up, a separate study of hybrid schemes is needed.

The paper is organized as follows. In Sec. 2 the iterative self-organized algorithm is suggested. In Sec. 3 the autoregressive phenomenological model of the stochastic 
iterative search process is proposed and the corresponding relations for secondorder statistics are derived. The model is used to parametrize MC data obtained for ferromagnetic $2 \mathrm{~d}$ Ising spin models on $L \times L$ square lattices (see Sec. (4).

\section{The iterations near the criticality}

We start by defining a process where temperature is driven by some response function. The stochastic process can be formally described by the second-order recursive formula

$$
\begin{aligned}
& T_{t+1}^{\mathrm{his}}=F_{T_{t}, N}, \\
& T_{t+1}=\eta T_{t+1}^{\mathrm{his}}+(1-\eta) T_{t} .
\end{aligned}
$$

It generates sequence $\left\{T_{t}, T_{t=0}=T_{0}, t=1,2, \ldots, N_{\text {event }}\right\}$. The stochastic function $F: T_{t} \rightarrow T_{t+1}^{\text {his }}$, which provides a preliminary histogrammatic estimate $T_{t+1}^{\text {his }}$ of unknown critical temperature $T^{*}$, is defined implicitly by

$$
F: \quad C_{T_{t+1}^{\text {his }}, T_{t}, N}=\max _{T \in\langle 0, \infty\rangle} C_{T, T_{t}, N} .
$$

The key point of the reweighting is to localize the extremal value of the response function $C$ evaluated for $N$ MC samples. Commonly, the response function can be defined by the first and second moments of energy or magnetization, respectively. The role of filtering, defined by the plasticity parameter $\eta$ in Eq. (31), is to weaken fluctuations 13 of the $T_{t+1}^{\text {his }}$ sequence.

Let us assume the invariant probability distribution function (pdf) of the form

$$
p(T)=\overline{\delta_{T, T_{t}}}=\lim _{t_{\max } \rightarrow \infty} \frac{1}{t_{\max }} \sum_{t=1}^{t_{\max }} \delta_{T, T_{t}},
$$

where $\delta$ is the Kronecker's symbol and $\cdots$ is the arithmetic average (which differs from standard thermodynamic average $\langle\ldots\rangle)$. From now on $T^{*}$ is associated with pseudocritical temperature $T_{\mathrm{c}}(L)$ of the specific heat. For Gaussian-like $p(T)$ we suppose

$$
\begin{aligned}
T^{*} & \simeq \lim _{t_{\max } \rightarrow \infty} \frac{1}{t_{\max }} \sum_{t=0}^{t_{\max }} T_{t}=\overline{T_{t}} \\
& =\int_{0}^{\infty} \mathrm{d} T p(T) T .
\end{aligned}
$$

As mentioned above, the specific heat can be estimated within a histogram reweighting approach using the fluctuation-dissipation relation

$$
C_{T, T_{t}}=\frac{\left\langle E^{2}\right\rangle_{T, T_{t}}-\langle E\rangle_{T, T_{t}}^{2}}{T^{2} L^{2}} .
$$

At the thermodynamic limit $N \rightarrow \infty$, the iterative process becomes deterministic and tends to an unique fixed point $T^{*}=F_{T^{*}, N \rightarrow \infty}$. For finite $N$, a convergence criterion have to be formulated in a pure statistical sense. The variance

$$
\mathcal{V}_{t}=\overline{\left(T_{t}-T^{*}\right)^{2}}
$$


has been chosen as a suitable measure that reflects the convergence.

\section{The model of the iteration process}

The iterative process, described by Eqs. (2), (3), (4) supplemented by Eq. (7), consists of nonlinear and stochastic rules. As an auxiliary tool of analysis, let us introduce a phenomenological model to parameterize the simulated statistics. Within the model, the explicit form of the stochastic function [see Eq. (2)] is

$$
F_{T_{t}, N}=\alpha T^{*}+(1-\alpha) T_{t}+\xi_{t},
$$

where an additive noise $\xi_{t}$ describes uncertainty. The convergence rate towards $T^{*}$ is determined by the parameter $\alpha$, which contains information of the systematic reweighting error. From Eqs. (2), (3) and Eq. (9) follows that

$$
T_{t+1}=\alpha \eta T^{*}+(1-\alpha \eta) T_{t}+\eta \xi_{t},
$$

where the noise term is controlled by the coefficient $\eta$. The model discussed here can be considered as an application of $\operatorname{ARMA}(1,1)$ model 14. The solution of Eq. (10) can be written simply as

$$
T_{t}=T^{*}+\left(T_{0}-T^{*}\right) B^{-t}+\eta B \sum_{q=0}^{t-1} \xi_{q} B^{q-t},
$$

where $B=1 /(1-\alpha \eta)$. The above formula, written for any $\xi_{t}$ dependence, is general. At this point, let us consider the statistical properties of Gaussian noise for $\xi_{t}$ specified by

$$
\begin{aligned}
\overline{\xi_{t}} & =0, \\
\overline{\xi_{t} \xi_{t^{\prime}}} & =A \delta_{t, t^{\prime}},
\end{aligned}
$$

where $A>0$. Within the limit of a second order statistics in $T_{t}$, from Eq. (11) and Eq. (12), follows

$$
\overline{T_{t}}=T^{*}+\left(T_{0}-T^{*}\right) B^{-t} .
$$

Therefore, the stationary regime is defined by the invariance $\overline{T_{t}}=T^{*}$, which stays in agreement with Eq. (6). If the solution given by Eq. (11) is substituted into Eq. (8), one obtains

$$
\mathcal{V}_{t}=B^{-2 t}\left(T_{0}-T^{*}\right)^{2}+\frac{A \eta^{2} B^{2}}{B^{2}-1}\left(1-B^{-2 t}\right),
$$

which is equivalent to the recursion $\mathcal{V}_{t+1}=A \eta^{2}+\mathcal{V}_{t} B^{-2}$. Terms proportional to $B^{-2 t}$ describe a transient regime of the initial temperature $T_{0}$, which the system forgets exponentially fast. Terms $\left(T_{0}-T^{*}\right)^{2} B^{-2 t}$ and $A \eta^{2} B^{2(1-t)} /\left(1-B^{2}\right)$ are deterministic and stochastic contribution to $\mathcal{V}_{t}$, respectively. The variance $\mathcal{V}_{t}$ remains finite for $t \rightarrow \infty$ if $B^{2}>1$, that is if $0<\alpha \eta<2$. The transient regime can be characterized by

$$
\mathcal{V}_{1}=A \eta^{2}+(1-\alpha \eta)^{2}\left(T_{0}-T^{*}\right)^{2}
$$


whereas, to obtain the stationary regime, one needs to calculate

$$
\mathcal{V}_{t \rightarrow \infty} \equiv \mathcal{V}_{\infty}=\frac{A \eta^{2} B^{2}}{B^{2}-1}=\frac{A \eta}{\alpha(2-\alpha \eta)} .
$$

It results that $\mathcal{V}_{\infty}$ diverges at $\alpha \rightarrow 2 / \eta$ or equivalently $B \rightarrow-1$. The characteristic transient time,

$$
\tau_{\operatorname{tr}}=-\frac{1}{\ln |1-\alpha \eta|}
$$

follows from its definition $\left(B^{2}\right)^{-t}=\exp \left(-2 t / \tau_{\text {tr }}\right)$. The transient time has a singularity for $\eta \rightarrow(1 / \alpha)^{ \pm}$and $B \rightarrow \pm \infty$. In this limit the model describes immediate convergence $\left(\tau_{\operatorname{tr}} \rightarrow 0\right)$ and variance $\mathcal{V}_{\infty}=A / \alpha^{2}$. A singular parametric line, $1-\alpha \eta=0$, splits region $B^{2}>1$ into alternating $(B<-1)$ and unidirectional convergence $(B>1)$ of $T_{t}$ near $T^{*}$.

Regarding the convergence properties, it is useful to introduce $\eta=\eta_{\mathrm{m}}$ as the value of the highest initial convergence rate, defined by the condition $\mathrm{d} \mathcal{V}_{1} /\left.\mathrm{d} \eta\right|_{\eta=\eta_{\mathrm{m}}}=$ 0 . This yields

$$
\eta_{\mathrm{m}}=\frac{\alpha\left(1-\frac{T_{0}}{T^{*}}\right)^{2}}{\frac{A}{\left(T^{*}\right)^{2}}+\alpha^{2}\left(1-\frac{T_{0}}{T^{*}}\right)^{2}} .
$$

For this extremal value is $\mathcal{V}_{1}\left(\eta_{\mathrm{m}}\right)=(A / \alpha) \eta_{\mathrm{m}}$.

Finally, we introduce some supplementary remarks about the limit of the vanishing error. Clearly, for $N \rightarrow \infty$ we assume $\alpha \rightarrow 1^{-}$. The consequence of Eqs. (17) and (18) is $\eta \rightarrow 0^{+}$, that implies $\mathcal{V}_{t \rightarrow \infty} \rightarrow 0$. Unfortunately, because of $\tau_{\mathrm{tr}} \rightarrow \infty$ this cannot be attained in practice.

\section{Numerical results}

In order to explore the proposed model by numerical simulation, we chose the $2 \mathrm{~d}$ Ising model with the Metropolis algorithm as testing ground. Most of the data are accumulated on square lattices of linear dimension $L=10$ and periodic boundary conditions. The methodology is expected to be quite general and of straightforward application for both discrete and continuous systems.

Every iteration step $t=1,2, \ldots, N_{\text {event }}$ of algorithm, based on Eq. (2) and Eq. (3), consists of the following main blocks (i)-(iv):

(i) equilibration that includes $10^{4}$ MCSS (Monte Carlo steps per spin) for constant $T_{t}$.

(ii) accumulation of $N$ pairs $\left(Q_{q}, E_{q}\right)$ into histograms. In order to reduce autocorrelations, successive samples are separated by one MCSS.

(iii) localization of the maximum $C_{T_{t+1}^{\mathrm{his}}, T_{t}, N}$ by a gradient algorithm. Recall, that $C$ is computed from Eq. (11) and Eq. (17) for $\langle E\rangle_{T, T_{t}}$ and $\left\langle E^{2}\right\rangle_{T, T_{t}}$. 
(iv) prediction of next $T_{t+1}$ using the filtering given by Eq. (3).

Fig. 1 illustrates the properties of transient regimes, which are affected by the choice of $T_{0}$ and $\eta$. The $\eta$-dependences of $\mathcal{V}_{1}$ are presented in Fig. 2(a). One may see that the agreement of the simulated data with Eq. (16) is quite satisfactory. Typical values of the parameters determined by the fit are gathered in Table 1

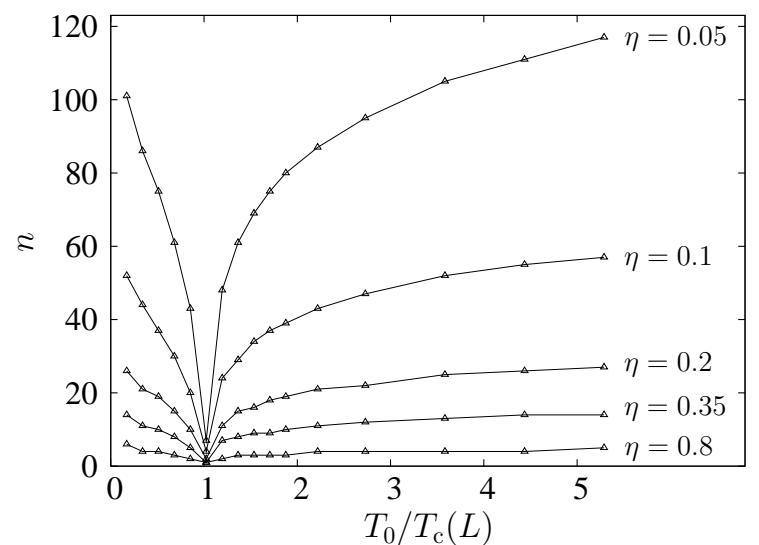

Fig. 1. The transient stages of the iterative regime characterized by the minimum number of iteration steps $n$ needed to satisfy the stop criterion $\left|T_{n}-T_{\mathrm{c}}(L)\right| \leq 0.01$ for resulting $T^{*}=$ $T_{\mathrm{c}}(L)=0.586141$. The number $n$ is plotted as a function of initial temperature $T_{0}$ [distinct from $\left.T_{\mathrm{C}}(L)\right]$ for several $\eta$. Calculated for fixed $N=10^{5}$.

Table 1. The transient regime parameters for $T_{0}=0.6$ obtained by fitting of Eq. (16).

\begin{tabular}{cccc}
$N$ & $\alpha$ & $A$ & $\eta_{\mathrm{m}}$ \\
\hline \hline $10^{3}$ & 1.01805 & $1.398 \cdot 10^{-4}$ & 0.5770 \\
$10^{4}$ & 1.00281 & $1.227 \cdot 10^{-5}$ & 0.9376 \\
$10^{5}$ & 0.99937 & $1.282 \cdot 10^{-6}$ & 0.9939 \\
$10^{6}$ & 0.99974 & $1.216 \cdot 10^{-7}$ & 0.9996
\end{tabular}

For $N \rightarrow \infty$, we observe that $\eta_{\mathrm{m}} \rightarrow 1^{-}$, which is in agreement with our expectations. The insert of Fig. 2(b) illustrates the dependence of $\eta_{\mathrm{m}}\left(T_{0}\right)$ with the special cases $T_{0}=T_{\mathrm{c}}(L)$ and $T_{0} \gg T_{\mathrm{c}}(L)$, which clearly coincide with Eq. (19). Additional numerical study reveals the discrepancy for $T_{0} \ll T_{\mathrm{c}}(L)$, that is caused by a non-trivial decrease of $\alpha$ by decreasing $T_{0}(\alpha<1)$. The $N$-dependence depicted in Fig. 2(b) is constructed for an initial value $T_{0}=0.6$. There is a certain $N$ value for which $\alpha=1$ and iterations cross from alternating to unidirectional convergence. In other words, statistical errors compensate systematic ones. In agreement with Ref. [8, we have verified statistical errors of MC averages to be proportional to $1 / \sqrt{N}$, which implies $A \sim 1 / N$. Actually, the fit provides $A \simeq 0.13 / N$. 

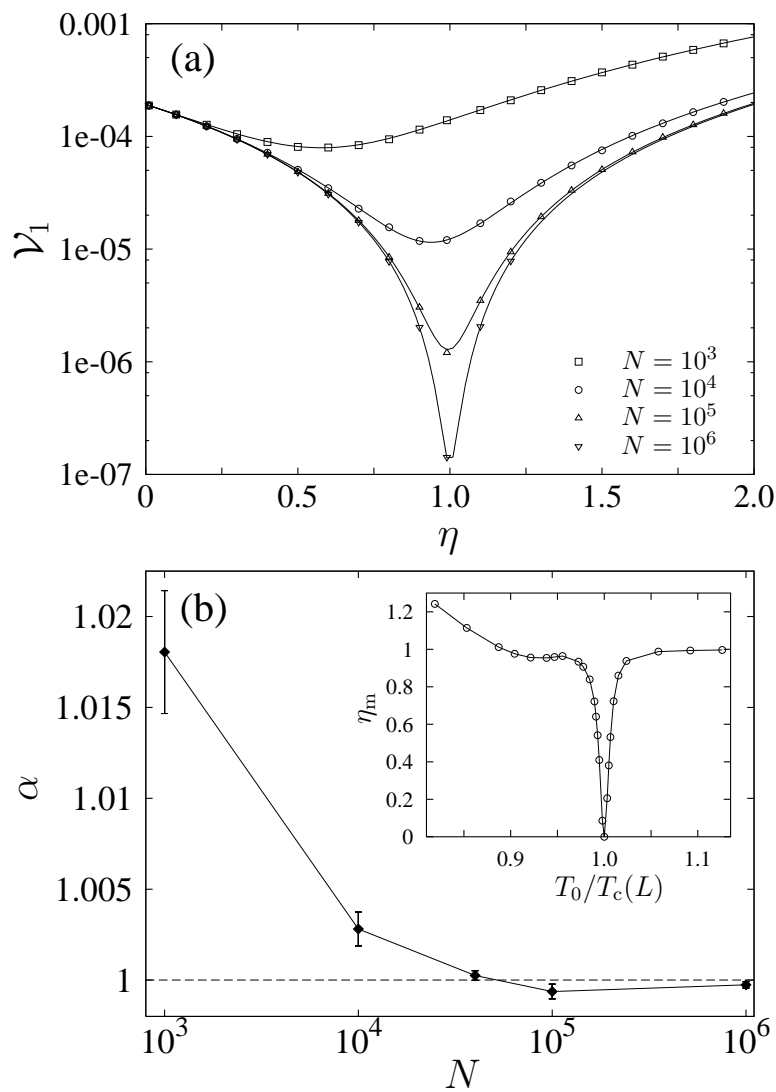

Fig. 2. The analysis of transient regime: (a) The $\eta$-dependence of variance $\mathcal{V}_{1}$ obtained for initial $T_{0}=0.6$. The lines correspond to the fits of $\eta$-dependences via Eq. 16. The parameters are collected in Table1 (b) The $\alpha(N)$ dependence for $T_{0}=0.6$. The inset depicts $\eta_{\mathrm{m}}\left(T_{0}\right)$ for $N=10^{4}$.

If the number of iterations is much larger than $\tau_{\text {tr }}$, the initial conditions are forgotten and thus invariant pdf's of temperature variable are formed (see inset in Fig. 3). The calculation of $T^{*} \equiv T_{\mathrm{c}}(L)\left(t \gg \tau_{\mathrm{tr}} \gg 1\right)$ is controlled by $\eta$. We have verified $\sqrt{\mathcal{V}_{t}} \propto \eta / \sqrt{N}$ for the stationary regime. The error increases monotonically with $\eta$ for a given $N$ as in Fig. B We have compared the simulation and analytical $\eta$-dependences of $\mathcal{V}_{\infty}$ for $A$ and $\alpha$, given in Table 1 and those determined by a fit of Eq. (17) for $N=10^{5}$. The comparison reveals a discrepancy of the $A$ parameter determination. The $A$ parameter, determined by the fit of Eq. (17) to numerical data, increases its value about $6.2 \times 10^{-8}$. A further extension of the model, e.g. taking into account a weak colored noise, should eliminate this distinction.

According to Eq. (6), the mean values of invariant pdf's (see inset in Fig. 3) were used to estimate $T_{\mathrm{c}}(10)=0.586141$. The method is straightforward applied for $L=10,20, \ldots, 100$. Subsequently, the values $T_{\mathrm{c}}(L)$ are interpolated by the finite size formula $T_{\mathrm{c}}(L)=T_{\mathrm{c}}+b / L$. This yields to estimate the thermal coeffi- 
cient $b=0.1889 \pm 0.0033$ and the critical temperature as $T_{\mathrm{c}}=0.5673 \pm 0.0001$. The last estimation stays in a satisfactory agreement with exact value $T_{\mathrm{c}}^{\mathrm{ex}}=$ $[2 \ln (1+\sqrt{2})]^{-1}=0.56729$. The problem of critical indices can be handled effectively in the frame of a two-lattice iterative algorithm, too 15. In that case, the objective function given by Eq. (4) has to be written in terms of the Binder cumulant, $F: U_{T_{t+1}^{\text {his }}, T_{t}, N}=\min _{T \in\langle 0, \infty\rangle}\left|U_{T, T_{t}, N}\left(L_{2}\right)-U_{T, T_{t}, N}\left(L_{1}\right)\right|$, for two different system sizes. According to the fact, that histogram reweighting allows a superior determination of the derivation $U_{T_{t+1}, T_{t}, N}^{\prime}(L) \equiv \mathrm{d} U_{T, T_{t}, N}(L) /\left.\mathrm{d} T\right|_{T=T_{t+1}}$, we considered, as an example, the exponent $\nu$ of the correlation length

$$
\left(\frac{1}{\nu}\right)_{t} \simeq \frac{\ln \left[\frac{U_{T_{t+1}, T_{t}, N}^{\prime}\left(L_{1}\right)}{U_{T_{t+1}, T_{t}, N}^{\prime}\left(L_{2}\right)}\right]}{\ln \left(L_{1} / L_{2}\right)} .
$$

Within the proposed algorithm, we generated a sequence of estimates (for each iteration step separately) of the critical exponent for the lattice pair $2 L_{1}=L_{2}=20$. Considering the peak of nonsymmetric pdf of $(1 / \nu)_{t}$ we determined $1 / \nu^{\text {ex }}=1$ with a relative error of $1.35 \%$.

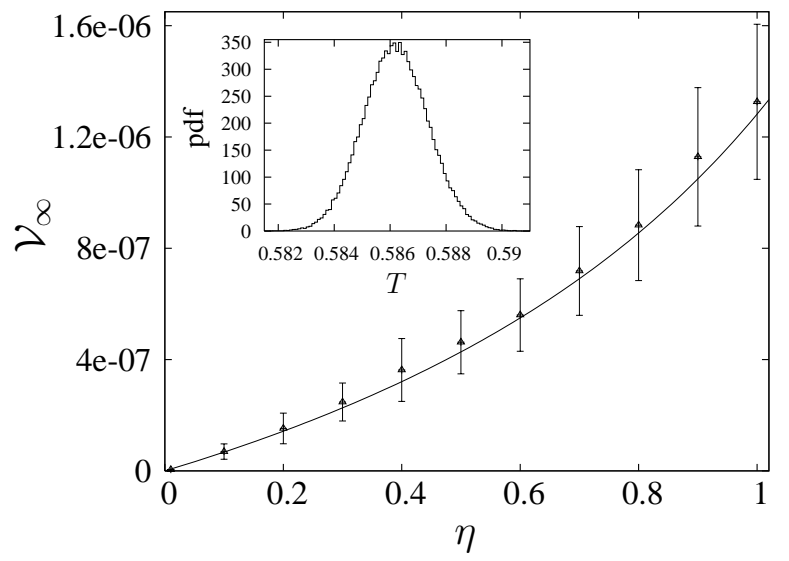

Fig. 3. The $\eta$-dependence of $\mathcal{V}_{\infty}$ compared with Eq. (17) for parameters $A, \alpha$ and $N=10^{5}$ given in Table 1 The error bars are calculated using 5000 independent runs. The inset depicts a stationary pdf of $T_{t}$ constructed for $N_{\text {event }}=10^{5}$ and $\eta=1$.

\section{Conclusions}

An iterative process that is useful around the critical point and includes Metropolis single spin-flip algorithm, single-histogram reweighting and linear filtering is studied numerically. The properties of this iterative algorithm are studied for the $2 \mathrm{~d}$ Ising ferromagnetic model on a square lattice. The total errors in the estimate of pseudocritical temperature are obtained. We demonstrated how the accuracy can be 
affected by $\eta$. However, one should be careful with an extremely small $\eta$ to avoid an enormous increase of transient time. Our analysis demonstrates that it is insufficient to use several reweighting trials to estimate total errors. The most interesting iterations are of this kind that compensate the systematic errors introduced by the histogram reweighting approach. Despite of the success of the linearized model, which allows interpretation of numerical data, further improvement of the proposed methodology is still expected. For instance, one should take into account non-linear corrections in Eq. (10) and/or the colored noise (that is, with autocorrelations of error terms) in Eq. (13). We believe that nontrivial dependences of $\alpha$ and $\eta_{\mathrm{m}}$ (see e.g. inset of Fig. 2(b)) could be further explained considering microscopic expression for the density of states 16 .

\section{Acknowledgments}

The authors would like to thank the Slovak Grant agency VEGA (grant No. 1/2009/05) and the agency APVT-51-052702 and the internal grant of the Faculty of Sciences of Šafárik University VVGS 2003 for financial support. The authors express their thanks to unknown referee for valuable corrections and suggestions.

\section{References}

1. K. Binder, D.W. Heermann, Monte Carlo Simulation in Statistical Physics (Springer, Berlin, 1998).

2. N. Metropolis, A.W. Rosenbluth, M.N. Rosenbluth, A.H. Teller, J. Chem. Phys. 21, 1087 (1953).

3. Z.W. Salsburg, J.D. Jacobson, W. Fickett, W.W. Wood, J. Chem. Phys. 30, 65 (1959); A.M. Ferrenberg, R.H. Swendsen, Phys. Rev. Lett. 61, 2635 (1988).

4. A.M. Ferrenberg, R.H. Swendsen, Phys. Rev. Lett. 63, 1195 (1989).

5. A.M. Ferrenberg, D.P. Landau, R.H. Swendsen, Phys. Rev. E 51, 5092 (1995).

6. A.M. Ferrenberg, D.P. Landau, Phys. Rev. B 44, 5081 (1991).

7. D.P. Landau, Journ. Magn. Magn. Mater. 200, 231 (1999).

8. E.P. Münger, M.A. Novotny, Phys. Rev. B 43, 5773 (1991).

9. L.P. Kadanoff, Physics Today (March 1991), p. 9.

10. H. Robbins, S. Munroe, Ann. Math. Stat. 22, 400 (1951).

11. N. Ito, G.A. Kohring, Int. J. Mod. Phys. C 5, 1 (1994).

12. J.A. Plascak, A.M. Ferenberg, D.P. Landau, Phys. Rev. E 65, 066702 (2002).

13. J.C. Principe, N.R. Euliano, W.C. Lefebvre, Neural and adaptive systems: Fundamentals through simulations (John Wiley \& Sons, Inc. 2000).

14. P.J. Brockwell, R.A. Davis, Introduction to Time Series and Forecasting (Springer, New York, 1996).

15. D. Horváth, M. Gmitra, Z. Kuscsik, Czech. J. Phys. 54, 921 (2004);

D. Horváth, M. Gmitra, Int. J. Mod. Phys. C 15, 1269 (2004).

16. P.D. Beale, Phys. Rev. Lett. 76, 78 (1997). 